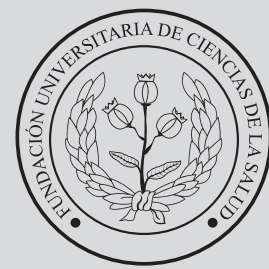

FUCS
Repr p

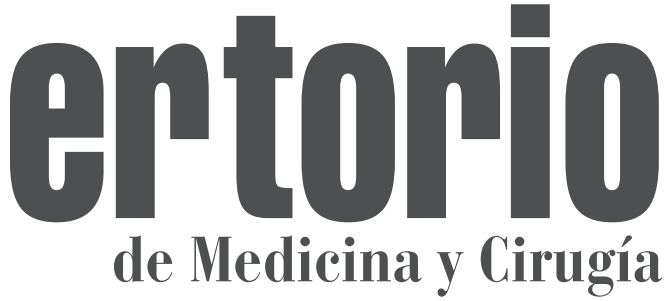

\title{
Estimación de la prevalencia del COVID-19 en Colombia
}

\section{Estimates of COVID-19 prevalence in Colombia}

angeniero. Magister en Gestión de la Tecnología Educativa, Especialista en Administración de la Informática Educativa. Docente de matemáticas e Investigador, Secretaría de Educación de Soacha, Cundinamarca.

\section{R E S U M EN}

Introducción: el nuevo Coronavirus (COVID-19) ha sido clasificado por la Organización Mundial de la Salud como una emergencia en salud pública de importancia internacional (ESPII). Se han reconocido casos en todos los continentes y el 6 de marzo se confirmó el primer caso en Colombia. Objetivo: estimar la medida de frecuencia de prevalencia en los departamentos de Colombia para COVID-19. Metodología: para desarrollar la investigación se utilizó la base de datos de las personas contagiadas por el COVID-19, la información de los datos corresponde al período acumulado a 31 de agosto 2020 para los departamentos de Colombia. Resultados: se estimó que a 31 de agosto 2020, la prevalencia para cada una de los departamentos de Colombia, se detalla que con mayor prevalencia de casos positivos por COVID-19, estuvieron: Amazonas 3,41\%, Bogotá 2,72\%, Atlántico 2,35\%, Caquetá 1,47\%, y Sucre 1,22\%; y en los departamentos con menor proporción: Vichada 0,00\%, Quindío 10,23\%, Casanare 0,24\%, Guainía 0,27\%, y Córdoba y Caldas 0,29\%. Conclusiones: se ha observado en lo corrido de 2020 un aumento paulatino de contagios por COVID-19 en Colombia hasta llegar a registrar a 31 de agosto 2020, 615.168 casos positivos. La tendencia probablemente seguirá aumentando hasta que no se encuentre una solución definitiva a la pandemia propiciada por el COVID-19.

Palabras clave: COVID-19, pandemia, prevalencia.

(C) 2020 Fundación Universitaria de Ciencias de la Salud - FUCS. Este es un artículo Open Access bajo la licencia CC BY-NC-ND (http://creativecommons.org/licenses/by-nc-nd/4.0/).

Historia del artículo:

Fecha recibido: septiembre 14 de 2020 Fecha aceptado: septiembre 18 de 2020

\section{INFORMACIÓN DEL ARTÍCULO}

Autor para correspondencia. Ing. Jorge Enrique Díaz Pinzón jediazp@unal.edu.co
DOI

10.31260/RepertMedCir.01217372.1115 
Introduction: the novel Coronavirus (COVID-19) has been declared by the World Health Organization as a public health emergency of international concern (PHEIC). Cases have been reported on all continents and the first case in Colombia was confirmed on March 6 2020. Objective: to estimate the measure of frequency of prevalence of COVID-19 cases for each department of Colombia. Methodology: the database including people infected with COVID-19 was used to develop the research, with the information accumulated up to August 312020 for the departments of Colombia. Results: by August 31 2020, the highest prevalence of COVID 19 positive cases among Colombia's departments was as follows: Amazonas $3.41 \%$, Bogotá $2.72 \%$, Atlántico $2.35 \%$, Caquetá $1.47 \%$, and Sucre $1.22 \%$; and the lowest prevalence was found in: Vichada $0.00 \%$, Quindío $10.23 \%$, Casanare $0.24 \%$, Guainía $0.27 \%$, and Cordoba and Caldas $0.29 \%$. Conclusions: There has been a gradual increase in the number of COVID-19 infections in Colombia, throughout year 2020, recording 615.168 positive cases by August 31 2020. The trend will probably continue to increase until a definitive solution to the COVID-19 pandemic is found.

Key words: COVID-19, pandemic, prevalence.

(C) 2020 Fundación Universitaria de Ciencias de la Salud - FUCS. This is an open access article under the CC BY-NC-ND license (http://creativecommons.org/licenses/by-nc-nd/4.0/).

\section{INTRODUCCIÓN}

La aparición del primer caso de neumonía por COVID-19 aconteció en Wuhan, China, en 2019. La investigación de 425 casos de la revista The New England Journal of Medicine reveló que los casos se exhibían en pacientes con una media de edad de 59 años; $56 \%$ eran masculinos y la agrupación epidemiológica era el mercado de animales vivos y mariscos. La fase de incubación promedio es de cinco días, con un rango entre cuatro y 14 días, así como una trasmisión de humano a humano, estimando que el R0 (velocidad con que una enfermedad puede propagarse) es de 2.2 a 3.7. La infección es a través de gotas o contacto estrecho con personas u objetos infectados con el virus. ${ }^{1}$ Al descubrir el COVID-19 se añadió como definición de caso el que hubieran estado en Wuhan o China 14 días anteriores o que fueran contacto de un caso dudoso o confirmado. El caso válido tiene una prueba positiva para COVID-19 por RT-PCR. ${ }^{2-4}$

Procedente de la evaluación firme del comportamiento del brote, el 30 de enero 2020 el Comité de Emergencias convocado por el Director General de la OMS en virtud del Reglamento Sanitario Internacional (RSI [2005]) se reunió y declaró al evento de COVID-19 como una emergencia de salud pública de categoría internacional (ESPII), y recomendó a todos los países estar dispuestos para adoptar medidas de contención, tales como el cuidado activo, la localización temprana, el aislamiento y el manejo de los casos, el rastreo de contactos y la prevención de la propagación del COVID-19. ${ }^{5}$

Ahora, en epidemiología, el desarrollo de investigación es análogo al utilizado en el resto de las ciencias. Cuando se investiga la salud de la población, asimismo se proponen una o varias explicaciones hipotéticas que posteriormente son reducidas a contrastación empírica. En este proceso, los conceptos de medición $\mathrm{y}$ de variable resultan primordiales. ${ }^{6}$ En epidemiología, las medidas de frecuencia de enfermedad más usualmente utilizadas se incluyen en dos categorías: prevalencia e incidencia. ${ }^{7}$

La prevalencia $(P)$ cuantifica la proporción de individuos de una población que padecen una enfermedad en un momento o periodo de tiempo determinado ${ }^{7,8,9}$. Su cálculo se estima mediante:

$$
\text { Prevalencia }=\frac{\begin{array}{c}
\text { No.de casos con la enfermedad } \\
\text { en un momento dado }
\end{array}}{\begin{array}{c}
\text { Total de población } \\
\text { en ese momento }
\end{array}} \times 100
$$

“Como todas las proporciones, la prevalencia no tiene dimensión y nunca toma valores menores de 0 a mayores de 1 , siendo frecuente expresarla en términos de porcentaje, en tanto por ciento, tanto por mil, en función de la "rareza" de la enfermedad estudiada". ${ }^{7}$

\section{RESULTADOS}

Cálculo de la prevalencia por contagio por COVID-19: para el cálculo de la prevalencia por contagio por COVID-19 se tuvo en cuenta la población por departamentos en Colombia con la información acumulada a 31 de agosto 2020, según la información registrada en el Instituto Nacional de Salud ${ }^{10}$ como se aprecia en la tabla 1. 


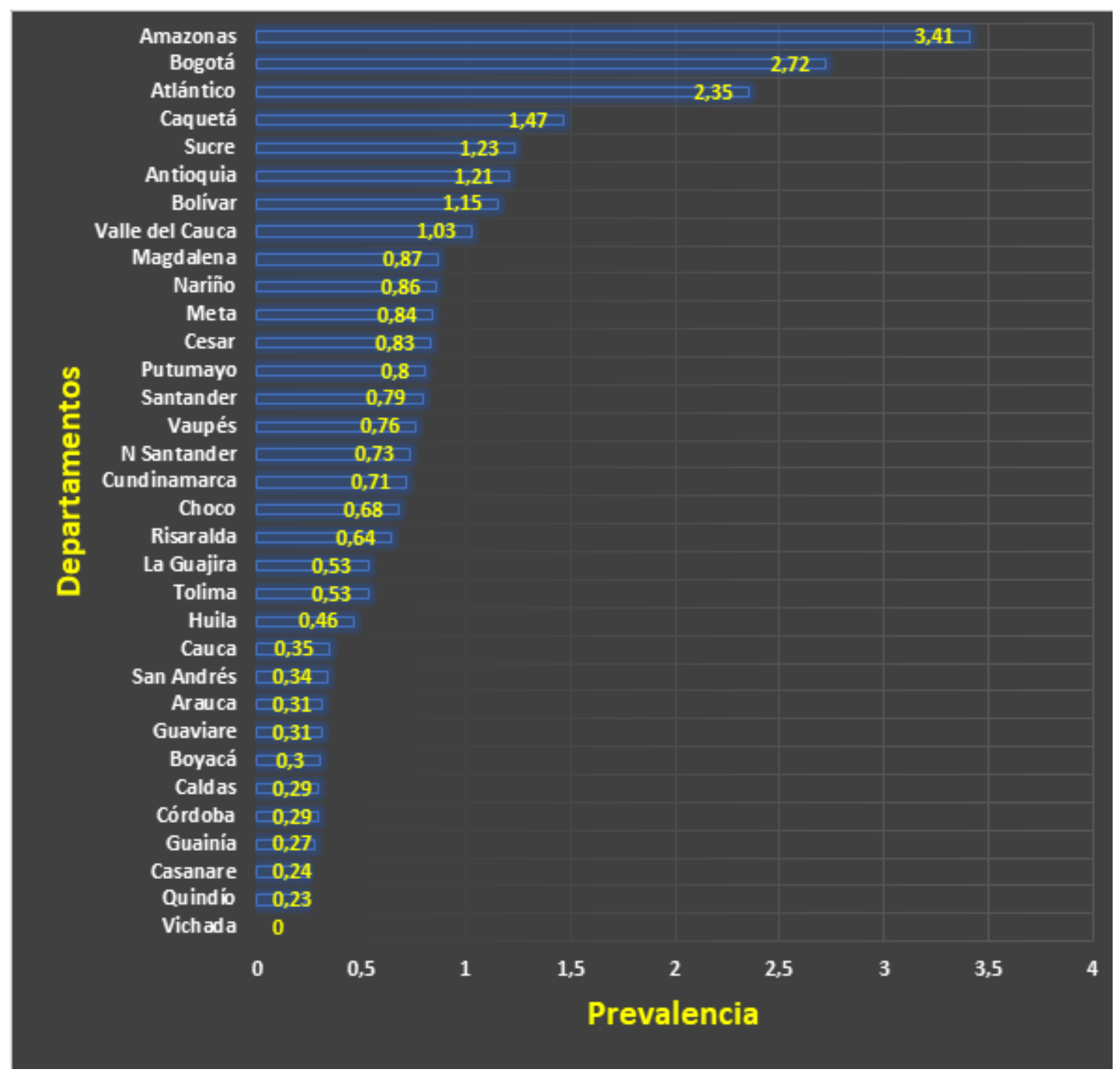

Figura 1. Prevalencia de contagio por COVID-19 por Departamentos en Colombia, a 31 de agosto 2020. Fuente: el autor.

En la figura 1 se aprecia los datos de la prevalencia en porcentaje $(\%)$, para cada uno de las departamentos de Colombia, se detalla que aquellos con mayor prevalencia de casos positivos por COVID-19, están: Amazonas 3,41\%, Bogotá 2,72\%, Atlántico 2,35\%, Caquetá $1,47 \%$, y Sucre $1,22 \%$; con menor proporción tenemos: Vichada 0,00\%, Quindío 10,23\%, Casanare 0,24\%, Guainía 0,27\%, y Córdoba y Caldas 0,29\%.

\section{CONCLUSIONES}

Se estimó a 31 de agosto 2020 la prevalencia para cada uno de los departamentos de Colombia se observa que la mayoría de casos positivos por COVID-19 fueron Amazonas 3,41\%, Bogotá 2,72\%, Atlántico 2,35\%, Caquetá 1,47\%, y Sucre $1,22 \%$; y con menor proporción tenemos: Vichada 0,00\%,
Quindío 10,23\%, Casanare 0,24\%, Guainía 0,27\%, y Córdoba y Caldas 0,29\%. En el caso de Bogotá, su prevalencia aumentó de acuerdo con el trabajo de investigación realizado por Díaz ${ }^{11}$ donde determinó para cada una de las localidades de Bogotá la prevalencia y mortalidad; en el total de Bogotá se evidenció una prevalencia de 2,23\%, con la información a 20 de agosto 2020 .

Se ha observado en lo corrido de 2020 un aumento paulatino de contagios por COVID-19 en Colombia, hasta llegar a registrar a 31 de agosto 2020, 615.168 casos positivos. La tendencia de casos positivos, probablemente seguirá aumentando hasta que no se encuentre una solución definitiva a la pandemia propiciada por el COVID-19.

Es importante estudiar la dinámica de posibles brotes infecciosos del COVID-19 en nuestro país, ya que esta enfermedad recientemente surgida ha tenido un rápido crecimiento de contagio. ${ }^{12}$ 
Tabla 1. Prevalencia de COVID-19 por departamentos. Colombia a 31 de agosto 2020

\begin{tabular}{|c|c|c|}
\hline Departamentos & Total de la población & Casos positivos \\
\hline Bogotá & 7743955 & 211300 \\
\hline Valle del Cauca & 4532152 & 46944 \\
\hline Atlántico & 2722128 & 64128 \\
\hline Amazonas & 79020 & 2701 \\
\hline Nariño & 1627589 & 14086 \\
\hline Antioquia & 6677930 & 80996 \\
\hline Cundinamarca & 3242999 & 23128 \\
\hline Meta & 1063454 & 8963 \\
\hline Chocó & 544764 & 3722 \\
\hline Cesar & 1295387 & 10815 \\
\hline Magdalena & 1427026 & 12460 \\
\hline Tolima & 1339998 & 7216 \\
\hline Bolívar & 2180976 & 25267 \\
\hline Risaralda & 961055 & 6225 \\
\hline Huila & 1122622 & 5167 \\
\hline Córdoba & 6677930 & 19738 \\
\hline Boyacá & 1242731 & 3824 \\
\hline Santander & 2280908 & 18211 \\
\hline Sucre & 949252 & 11709 \\
\hline Caldas & 1018453 & 2964 \\
\hline Cauca & 1491937 & 5285 \\
\hline N Santander & 1620318 & 11850 \\
\hline Quindío & 555401 & 1316 \\
\hline La Guajira & 965718 & 5139 \\
\hline Casanare & 435195 & 1060 \\
\hline Vaupés & 44712 & 340 \\
\hline Caquetá & 410521 & 6067 \\
\hline San Andrés & 63692 & 219 \\
\hline Putumayo & 359127 & 2895 \\
\hline Guainía & 50636 & 140 \\
\hline Guaviare & 86657 & 273 \\
\hline Arauca & 294206 & 925 \\
\hline Vichada & 112958 & 1 \\
\hline
\end{tabular}

Fuente: el autor.

\section{REFERENCIAS}

1. Hernández Orozco HG, Ramiro Mendoza MS, Trejo González R. ¿Cuáles son las medidas de prevención contra el Novel Coronavirus (COVID-19)? Rev Latin Infect Pediatr. 2020;33(1):4-6. DOI: $10.35366 / 92380$.
2. Secretaría de Salud, Subsecretaría de Prevención y Promoción de la Salud. Lineamiento estandarizado para la vigilancia epidemiológica y por laboratorio de enfermedad por 2019-COVID19. México: Formación editorial: LDG. Brenda Liliana Escobedo López; 2020. p. 60.

3. Comite Nacional para la Vigilancia Epidemiológica, Unidad de inteligencia Epidemiológica y Sanitaria. Enfermedad COVID-19 por SARS-CoV-2. Aviso epidemiológico CONAVE/04/2020/2019. México. 2020. p. 5.

4. Unidad de inteligencia Epidemiológica y Sanitaria. Comunicado técnico diario nuevo coronavirus en el mundo (2019-COVID19) 25/02/2019. México: Unidad de inteligencia Epidemiológica y Sanitaria 2020. p. 2.

5. World Health Organization. Statement on the first meeting of the International Health Regulations (2005) Emergency Committee regarding the outbreak of novel coronavirus (2019$\mathrm{nCoV}$ ) [Internet]. World Health Organization; 2020 [cited 2020 julio 21]; Available from: https://www.who.int/news-room/ detail/23-01-2020-statement-on-the-meeting-of-the-internationalhealth-regulations-(2005)-emergency-committee-regarding-theoutbreak-of-novel-coronavirus-(2019-ncov).

6. Moreno-Altamirano A, López-Moreno MC, Sergio , CorchoBerdugo A. Principales medidas en epidemiología. Salud Pública de México. 2000;42(4):337-48.

7. Pita Fernández S, Pértegas Díaz S, Valdés Cañedo F. Medidas de frecuencia de enfermedad [Internet]. España: Elsevier; 2004 [citado 2020 julio 21]; Disponible en: https://www.fisterra. com/formacion/metodologia-investigacion/medidas-frecuenciaenfermedad/.

8. Pinto A. Prevalencia e Incidencia [Internet]. Slideshare; 2014 [citado 2020 agosto 21]; Disponible en: https://es.slideshare.net/ alexpinto18/prevalencia-e-incidencia-2.

9. Quintana-Salgado L. Medidas de frecuencia en epidemiología [Internet]. 2015 [citado 2020 julio 21]; Disponible en: https:// es.slideshare.net/lualberts20/medidas-de-frecuencia-enepidemiologa-2015.

10. Instituto Nacional de Salud. COVID-19 en Colombia [Internet]. Colombia: Instituto Nacional de Salud; 2020 [citado 2020 agosto 31]; Disponible en: https://www.ins.gov.co/Noticias/Paginas/ Coronavirus.aspx.

11. Díaz Pinzón JE. Medidas de frecuencia por COVID-19 en Bogotá DC. Repert Med Cir. 2020;29(Núm. Supl.1):94-8. DOI: https://doi. org/10.31260/RepertMedCir.01217372.1110

12. Díaz Pinzón JE. Proyección del COVID-19 en Colombia. Rev Med. 2020;28(1):11-20. DOI: https://doi.org/10.18359/rmed.4702 